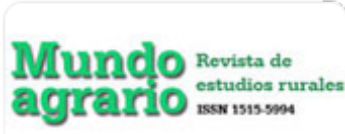

Mundo Agrario

ISSN: 1515-5994

mundoagrario@fahce.unlp.edu.ar

Universidad Nacional de La Plata

Argentina

\title{
La defensa de los recursos forestales: el movimiento social en la comunidad de Cherán, Michoacán, México
}

Cruz Coria, Eika; Zizumbo Villarreal, Lilia; Velázquez Castro, Judith Alejandra

La defensa de los recursos forestales: el movimiento social en la comunidad de Cherán, Michoacán, México

Mundo Agrario, vol. 21, núm. 46, 2020

Universidad Nacional de La Plata, Argentina

Disponible en: http://www.redalyc.org/articulo.oa?id=84562590007

DOI: https://doi.org/10.24215/15155994e138

Esta obra está bajo una Licencia Creative Commons Atribución-NoComercial-Compartirlgual 4.0 Internacional. 
Artículos

\section{La defensa de los recursos forestales: el movimiento social en la comunidad de Cherán, Michoacán, México}

Forest Resources Defense: the social movement in the community of Cherán, Michoacán, México

Eika Cruz Coria

Universidad Autónoma del Estado de Hidalgo, México

ecoria84@hotmail.com

Lilia Zizumbo Villarreal

Universidad Autónoma del Estado de México, México

lzv04@yahoo.com.mx

Judith Alejandra Velázquez Castro

Universidad Autónoma del Estado de Hidalgo, México

judithalejandra666@gmail.com

\author{
DOI: https://doi.org/10.24215/15155994e138 \\ Redalyc: http://www.redalyc.org/articulo.oa?id=84562590007
}

Recepción: 04 Junio 2019

Aprobación: 21 Octubre 2019

\section{Resumen:}

El objetivo de este estudio es analizar la estructura organizativa del movimiento social (MS) emprendido por la comunidad de Cherán, Michoacán, para la defensa de sus recursos forestales ante el saqueo orquestado por talamontes vinculados con la delincuencia organizada. Este movimiento fue analizado a la luz de la teoría de movilización de recursos (TMR). Para la recolección de datos, se aplicaron cuarenta entrevistas semiestructuradas a miembros de las organizaciones y otros actores que participaron en el movimiento. La formalización de las estructuras tradicionales de organización y la movilización del derecho fueron las dos fuerzas principales que dieron lugar al reconocimiento a la libre determinación de esta comunidad.

Palabras clave: JEL Q34 movimiento social, JEL Q23 recursos forestales, JEL Q58 oportunidades políticas, JEL P32 organización social, JEL P37 talamontes.

\section{Abstract:}

The objective of this study is to analyze the social movement (SM) in the community of Cherán, Michoacán, to uphold their forest resources against illegal logging linked to organized crime. This paper focuses on the internal organizational structure of the SM. The Resource Mobilization Theory (RMT) was used as a theoretical and methodological tool to analyze such social movement. Forty semi-structured interviews were conducted with community members and other social actors who participated in the movement. The formalization of traditional organizational structures and legal recourse were the two main factors in the recognition of the community self-determination.

KEYWORDS: JEL Q34 social movement, JEL Q23 forest resources, JEL Q58 political opportunities, s JEL P32 social organization, JEL P37 loggers.

\section{INTRODUCCIÓN}

De acuerdo con algunos autores, la exportación de recursos naturales sin mayor valor agregado a manera de commodities se ha intensificado durante las últimas décadas en los países de América Latina (Galafassi, 2011; Composto, 2012; Svampa, 2013). En México, el avance del capitalismo a partir de la explotación de la naturaleza se hace cada vez más patente sobre los bienes que aún mantienen su condición de comunes. La oleada extractivista basada en la comodificación de la naturaleza incluye actividades como la explotación petrolífera, la minería a cielo abierto, los agronegocios basados en transgénicos, y la expansión de la frontera forestal, entre otras actividades. Esta situación ha colocado al país en el nuevo orden geopolítico, pero también 
lo sitúa frente a una serie de asimetrías económicas, sociales, políticas y ambientales (Svampa, 2012; Lang, 2012).

Los recursos forestales vinculados a las comunidades rurales no escapan a este boom mundial por las materias primas y la producción de bienes y servicios. Varios autores han documentado un importante deterioro de la cobertura forestal del país a raíz de la expansión de las industrias de madera y de celulosa para cubrir la demanda internacional de papel, las concesiones mineras, la construcción de megaproyectos hidroeléctricos y el desarrollo de la ganadería extensiva en áreas forestales (Forlani, 2014; León, 2015).

De acuerdo con el Consejo Civil Mexicano para la Silvicultura Sostenible (CCMSS), México cuenta con 64,5 millones de hectáreas de bosques y selvas, de los cuales el 22,56\% se encuentra concesionado para el desarrollo de actividades mineras. Se han documentado algunas disputas entre empresas/proyectos mineros y comunidades, entre ellas: el conflicto entre pueblo Wixárika en el estado de San Luis Potosí y la minera canadiense First Majestic Silver Corp.; la pugna entre la comunidad de Nuevo Balsas en el estado de Guerrero y la empresa Torex Gold (Ortíz y Madrid, 2017), y el conflicto entre la empresa TransCanada y las comunidades rarámuris del estado de Chihuahua por la extracción del gas shale y la construcción de gaseoductos para su transporte, lo cual ha generado una tala inmoderada de árboles en la región (Tourliere, 2017).

En este contexto, las comunidades indígenas y los núcleos agrarios no solo han tenido que hacer frente a la embestida del capital trasnacional y al interés del estado por la explotación de sus recursos forestales, sino que también se enfrentan a la presencia de otros actores sociales -tales como el crimen organizado, cazadores furtivos, talamontes, empresas trasnacionales-, que en alianza con la clase política de los distintos niveles de gobierno, han comenzado a disputarse tanto los territorios como la riqueza natural que albergan.

El disímil lenguaje de valoración e intereses divergentes entre los actores sociales por la apropiación de los recursos naturales ha colocado a los conflictos socioambientales en la escena de la conservación, lo cual está vinculado -entre otras cosas- al (re)surgimiento de (nuevas) formas de participación social a favor de la defensa de los bienes naturales. Las comunidades indígenas y campesinas han alcanzado cierta notoriedad en lo que respecta a la conservación de la biodiversidad. Su participación en movimientos sociales (MS) asociados a la defensa de los recursos naturales obedece a la serie de agravios directos que estos "nuevos" actores sociales ejercen sobre sus territorios.

En este contexto, las comunidades se han constituido como actores político-ecológicos emergentes que actúan, generalmente, respaldados por una densa red multiescalar de actores sociales, y por los incentivos, las aperturas institucionales, las élites, los instrumentos técnicos y legales, y todo aquello que funciona como recursos y oportunidades políticas para la expansión de los movimientos sociales.

El objetivo de este trabajo es analizar el movimiento social (MS) emprendido por la comunidad indígena de San Francisco de Cherán, Michoacán, en defensa de sus recursos forestalesante el saqueo por parte de grupos de talamontes vinculados con la delincuencia organizada. Se enfatiza la movilización social y política y el uso del derecho como los principales recursos que dieron lugar a la creación de un proyecto político más amplio, que incluye la recuperación del control social del territorio y el reconocimiento a la libre determinación de este pueblo indígena.

Entre los diversos trabajos académicos enfocados en el estudio del MS gestado por esta comunidad se identifican aquellos que enfatizan en el ejercicio del poder indígena (Santillán, 2014), en el esquema de democracia interna (Aragón, 2013; Martínez, 2017), en las formas de organización, y en la autonomía indígena (Calveiro, 2014; Mandujano, 2014; Ojeda, 2015) como los factores que permitieron la construcción de un proyecto colectivo de resistencia en esta comunidad.

También se identifican otros trabajos que, situándose en el plano político-jurídico, analizan el uso del derecho internacional como una de las principales estrategias legales utilizadas por las organizaciones comunitarias de Cherán para defender su derecho a la autodeterminación (Ventura, 2012; Aragón, 2013, 2017; González y Zertuche, 2017). Y finalmente otras aportaciones que, si bien escapan a estas dos grandes 
tendencias, abordan dicho movimiento desde la praxis educativa, la seguridad o la conservación de los recursos forestales (Velázquez y Lepe, 2013; Marín y Ramírez, 2014; España y Champo, 2016).

Como se observa, son escasas o nulas las investigaciones que señalan a la estructura organizativa del movimiento que explica la consolidación de formas alternativas de resistencia en los pueblos indígenas. Este trabajo ofrece una explicación desde dicha postura, para ello se apoya en la teoría de la movilización de recursos (Tilly, 1978; Tarrow, 2002) y en las aportaciones realizadas por Favela y Murillo (2002) en relación a las oportunidades políticas que dan cauce o limitan la movilización de recursos en un MS. Se trata de una investigación de tipo cualitativa que se apoyó en historias de vida y en entrevistas en profundidad como principales instrumentos de recolección de información.

\section{LOS MOVIMIENTOS SOCIALES: FUNDAMENTO TEÓRICO}

A partir de los años sesenta se suscitaron una serie de movimientos sociales (MS) cuyas características diferían de los que se habían producido en épocas anteriores en diversas partes del mundo. Tras el insuficiente poder explicativo del modelo marxista y el estructural-funcionalista, surgen en Europa y Estados Unidos las teorías europeas sobre los nuevos movimientos sociales y el enfoque de movilización de recursos respectivamente. Ambas perspectivas encontraron en la teoría de la acción colectiva una nueva forma de interpretación de los movimientos sociales modernos (Riechman y Fernández, 1995; Berrío, 2006).

Este trabajo de investigación retoma la teoría de la movilización de recursos (TMR) (Oberschall, 1973; Gamson, 1975; McCarthy y Zald, 1977; Tilly, 1978, Tarrow, 2002) por su capacidad explicativa sobre los recursos, las formas organizativas y las oportunidades políticas que dan cauce a los movimientos sociales, y las aportaciones realizadas por Favela y Murillo (2002) en relación al aprovechamiento de las oportunidades políticas que ofrece el entorno. Bajo esta propuesta teórica, los MS se conceptualizan como "grupos racionalmente organizados que persiguen determinados fines y cuyo surgimiento depende de los recursos organizativos de que disponen" (Laraña, 1999, p. 15). A diferencia de otros abordajes, esta teoría considera la movilización colectiva como una forma de acción racional organizada ${ }^{1}$. Aunque en algunas ocasiones la reivindicación colectiva se presenta como una acción individual, esporádica, poco integrada y con fronteras difusas, para esta propuesta los MS son un proceso continuo de acción colectiva determinado por diferentes niveles de organización (formal e informal) (Ramírez, 2016).

En este sentido, la teoría se centra en conocer los elementos que desencadenan, permiten el desarrollo y el fortalecimiento o el fracaso de la acción colectiva, a fin de comprender mejor los patrones de movilización y predecir cuáles facilitan su consolidación y cuáles no (Martí, 2004). Para algunos autores, este planteamiento es un modelo multifuncional que propone el análisis integral de las condiciones estructurales que explican un MS, tales como: la disponibilidad de los recursos, , la organización y las oportunidades políticas (McCarthy y Zald, 1977; Berrío, 2006).

De acuerdo con Craig (1994), los recursos son aquellas ventajas tangibles (como dinero, locales, tierra, etc.) e intangibles (conocimientos técnicos, relaciones, conocimientos, etc.) que son seleccionadas por el grupo y dirigidas hacia el objetivo del cambio social. Esta propuesta teórica asume la existencia de dos vías desde las cuales provienen los recursos movilizados: de los beneficiarios directos del MS y de fuentes institucionales (fundaciones privadas, instituciones de asistencia social, medios de comunicación de masas, universidades, agencias gubernamentales y corporaciones empresariales).

La movilización de los recursos a favor de una acción colectiva es improbable sin la existencia de una infraestructura organizativa capaz de encauzarlos hacia el objetivo del movimiento. Para Mc Adam, Mc Carthy y Zald, la infraestructura organizativa está conformada por "los canales colectivos tanto formales como informales a través de los cuales la gente puede movilizarse e implicarse en la acción colectiva" (1999, p. 24). Si se admite esta premisa, entonces se asume que las estructuras de movilización no solo se encuentran 
relacionadas con los recursos, sino también con la agregación de intereses, la acción colectiva, su potencial de movilización y con las oportunidades políticas del entorno.

Para la TMR, un movimiento fluye por la presencia de un "núcleo duro" (March y Olsen, 1989, p. 68), que esta formado por grupos de nivel medio, organizaciones y redes formales como elementos significativos que dan lugar al surgimiento, mantenimiento e impacto de estas formas de acción colectiva (Fernández y Antolín, 2000). Para algunos autores (Tilly, 1978; Ferree, 1994; Mc Adam, Mc Carthy y Zald, 1999), el potencial de un movimiento está determinado por la presencia de grupos con identidades fuertes y redes interpersonales densas, exclusivas de los miembros del movimiento. Otros consideran la necesidad de un entorno organizativo que otorgue densidad al núcleo, es decir, se requiere del apoyo de redes extralocales de nivel meso y macro, las cuales también juegan un rol importante debido a que hacen posible la aparición de organizaciones lo suficientemente firmes como para resistir a los oponentes (Martí, 2004; Puricelli, 2005).

Así como la estructura organizativa es capaz de movilizar los recursos a favor del movimiento, también puede hacerlo la estructura de las oportunidades políticas. Se entiende por oportunidad política a "las dimensiones consistentes del entorno político, que fomentan o desintegran la acción colectiva de la gente" (Tarrow, 1997, p. 235). Para Favela y Murillo (2002), los MS no solo están determinados por las circunstancias del entorno político, sino también por las instituciones políticas del estado donde se desarrolla. Por tanto, se debe atender a los elementos estructurales (distribución del poder, sistema electoral, estructura de la representación de intereses legislación e instituciones que vigilan y controlan las protestas) y a los de tipo coyuntural (disponibilidad de aliados, alineamientos electorales, cohesión de élite, estrategias de resolución de conflictos) para comprender las estrategias políticas utilizadas por el propio movimiento. Aunque la estructura de las oportunidades políticas es regularmente un concepto que alude al contexto macro de un movimiento, en este trabajo también se considera que en el interior de un movimiento -desde una perspectiva microsociológica- las oportunidades políticas son recursos que incentivan la movilización de los individuos para actuar a favor de la consecución de los bienes colectivos (Craig, 1994; Pizzi y Brunet, 2012).

El estudio de las relaciones existentes entre la estructura organizativa de un MS, los recursos y el aprovechamiento de las oportunidades políticas permite la generación de conocimiento en torno a los principios de regularidad en los patrones de la acción colectiva pero, más aún, el análisis de los MS desde los elementos propuestos permite dar cuenta de que un movimiento: a) surge por la existencia de redes solidarias preexistentes a través de las cuales se canalizan rápidamente las tensiones emergentes; estas redes, a su vez, actúan como cauces por los que se puede iniciar la movilización, y b) debe tener recursos materiales y organizativos suficientes y adecuados para hacer frente a la oposición. No obstante, también es esencial comprender que un MS está influido por los elementos relativamente variables del ambiente político, elementos que pueden ser utilizados por la propia estructura organizativa del movimiento para alcanzar sus objetivos.

\section{Consideraciones Metodológicas}

Cherán es un municipio localizado en el noroeste del estado de Michoacán, en la región de la Meseta Purépecha (ver Figura 1). Esta comunidad indígena está integrada por cuatro barrios (en idioma purépecha Karhákua, Jarhukutini, Ketsikua y Paríkutini) rodeados por serranías que forman parte del sistema volcánico transversal. Su territorio tiene extensos bosques de pino y encino, y es una zona de infiltración de agua que recarga los mantos acuíferos que alimentan los cultivos de maíz y aguacate de la región. 
FIGURA 1

Ubicación del municipio de Cherán, Michoacán, México

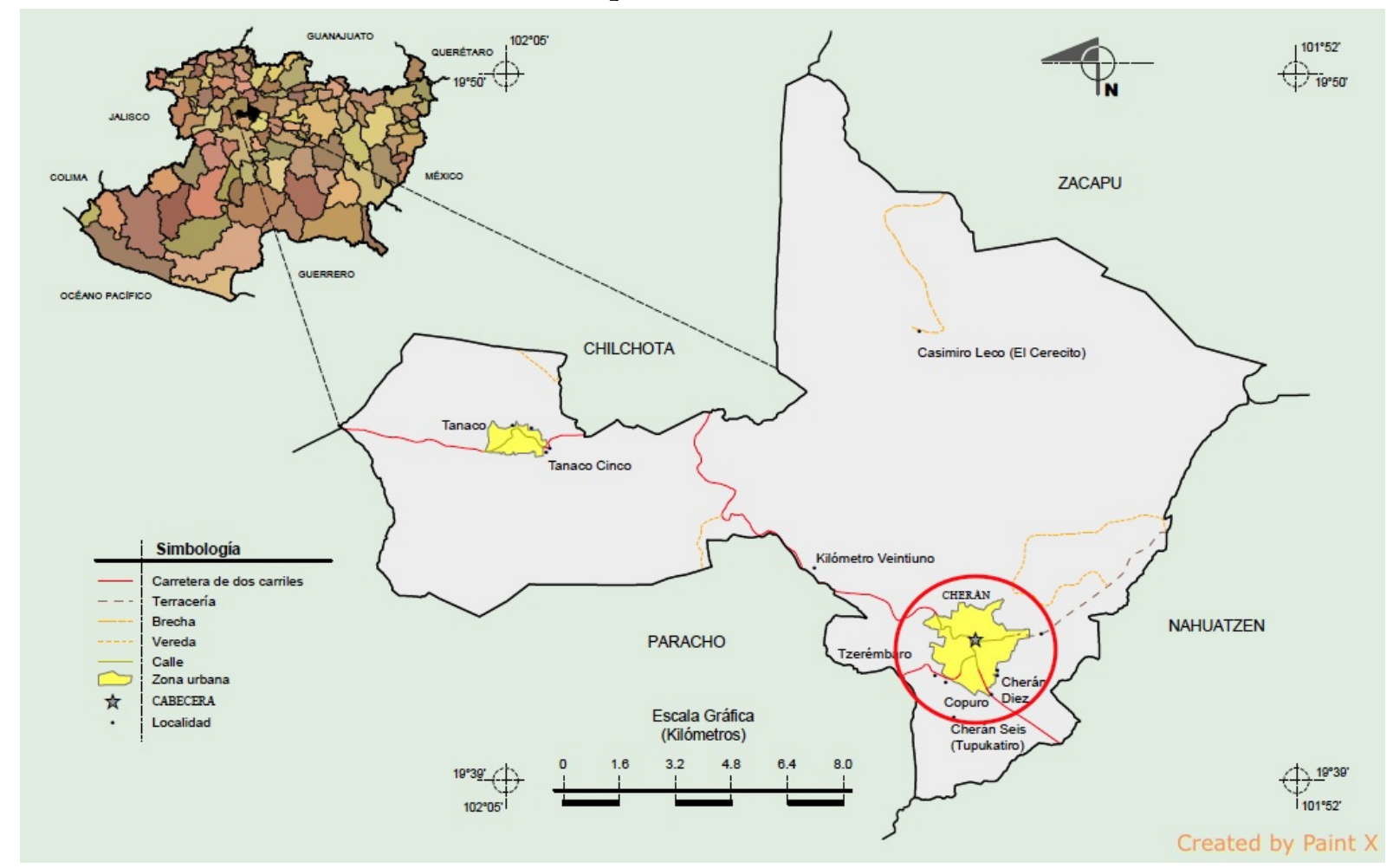

Instituto Nacional de Estadística y Geografía, 2010.

El territorio ocupado por este municipio abarca 21.170,21 hectáreas, de las cuales 16.684 son terrenos forestales de propiedad comunal (Secretaría de Medio Ambiente y Recursos Naturales, 2017). Solo un año antes del proceso intensivo de devastación del bosque a manos de talamontes (2008) se estimaba que el total de la cobertura forestal del territorio -ocupado por pino, encino, roble y oyamel-ascendía a 9.134 ha, y la destinada a programas de manejo forestal sustentable ocupaba un total de 7.237,59 ha (Instituto Nacional de Estadística y Geografía, 2007).

Para el estudio del MS generado en este territorio se retomó la teoría de la movilización de recursos (TMR) (Oberschall, 1973; Gamson, 1975; McCarthy y, Zald, 1977; Tilly, 1978, Tarrow, 2002) y las aportaciones realizadas por Favela y Murillo (2002) en relación a las oportunidades políticas. Así, se propuso el análisis integral de la disponibilidad de los recursos, los canales colectivos formales e informales y las oportunidades políticas en el entorno que dieron lugar al movimiento de Cherán (ver Figura 2). Desde el punto de vista cualitativo, este movimiento fue analizado en su integralidad a partir de dichas dimensiones. No obstante, partiendo de la idea de que un MS no es un fenómeno social estático ni lineal, y de que su análisis tiene elementos dinámicos, se retomaron las nociones de recursos, oportunidades políticas y formas de organización como elementos que atraviesan tres momentos que conforman el movimiento de Cherán: la visibilidad, el desarrollo y la movilización del derecho. 
FIGURA 2

Elementos metodológicos de análisis en el movimiento social de Cherán

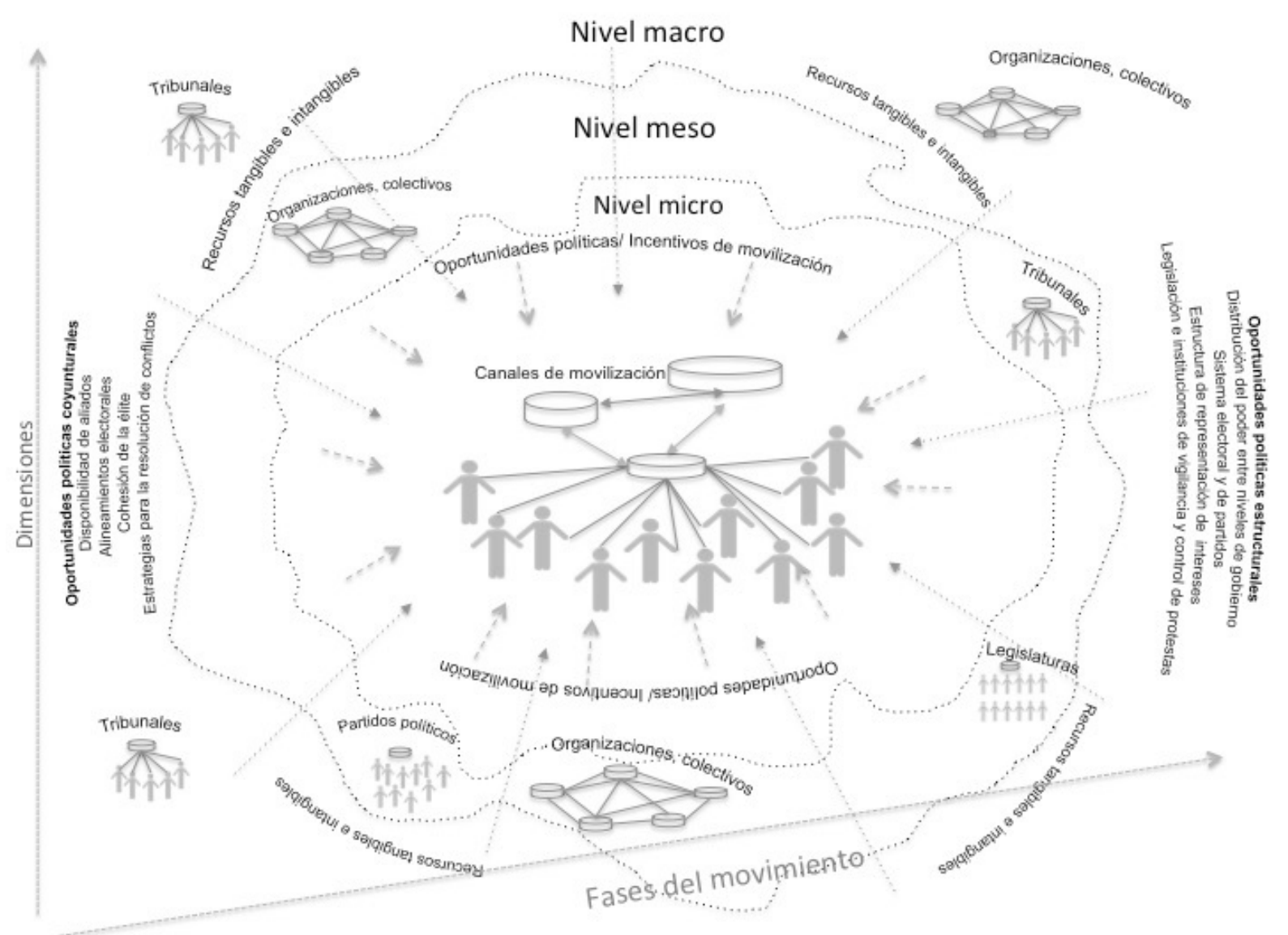

Elaboración propia a partir de Oberschall, 1973; Gamson, 1975; McCarthy y Zald, 1977; Tilly, 1978; Favela y Murillo, 2002.

Para la reconstrucción de las estructuras organizativas fueron entrevistados diversos actores comunitarios y otros que participaron en alguna o en todas las fases del movimiento. Para identificar los diferentes niveles de organización interna del MS, inicialmente se entrevistó a quienes fungieron como presidente y tesorero de la Asamblea General de Comuneros (AGC) y a tres (ex)integrantes de la Coordinación General del Movimiento de Lucha (CGML). También fueron entrevistados cuatro (ex)habitantes de cada uno de los barrios del municipio, que participaron en las organizaciones destinadas a la protección del territorio: barricadas, las fogatas y la ronda comunitaria. Adicionalmente, se aplicó un muestreo de bola de nieve para identificar a los actores sociales que incidieron en el estallido del movimiento; se entrevistaron siete mujeres y cinco hombres.

En esta etapa se utilizó una guía de entrevista semiestructurada, diseñada para obtener información con relación a: 1) el proceso de conformación del movimiento (fase de visibilidad), recursos movilizados y estructuras informales de organización; 2) el desarrollo del movimiento, estructuras formales de organización y oportunidades políticas en términos de los incentivos individuales. En una segunda fase se realizaron entrevistas a (ex)representantes -en su mayoría- de las organizaciones a nivel regional y nacional que constituyeron las redes extralocales de apoyo al movimiento: Acción Directa Autogestiva (ADA)-Puebla, Frente de Pueblos en Defensa de la Tierra y Agua Puebla- Tlaxcala (FPDTAPT), Alianza Mexicana de Organizaciones Sociales (AMOS), Centro de Derechos Humanos Tlachinollan. A nivel internacional, se obtuvo respuesta vía correo electrónico de dos representantes de organizaciones internacionales: Organización de Inquilinos de Cochabamba-OINCO (Bolivia) y Plataforma de Solidaridad con Chiapas 
y Guatemala de Madrid (España). También fueron entrevistados dos de los abogados-activistas, que acompañaron al CGML en el proceso legal que se entabló en defensa de su autonomía. En total se aplicaron 40 entrevistas. El trabajo de campo se realizó entre los meses de enero y agosto de 2017. Para el análisis de la información, se utilizó el método interpretativo, a fin de elaborar una descripción ideográfica que permitiera comprender la conducta de las figuras involucradas en el movimiento y la organización del mismo.

\section{Resultados del estudio de campo. Presentación y análisis}

En este apartado se abordan las tres fases del movimiento, que fueron identificadas durante el trabajo de campo: la visibilidad, el desarrollo y la movilización del derecho. En la primera sección se describen el cambio en el control y la distribución de los recursos forestales como los hechos que dieron lugar a la movilización de la población de Cherán en contra de los talamontes vinculados con el crimen organizado. En la segunda se presentan los canales formales e informales, así como las redes extralocales que dieron cauce a la movilización social y política y a la recuperación del territorio. Finalmente, se aborda la movilización del derecho como el recurso que permitió emprender un activismo político a favor del reconocimiento de la autodeterminación de Cherán como pueblo indígena.

\subsection{La fase de visibilidad: las organizaciones informales}

Históricamente, la riqueza forestal de esta comunidad la colocó en medio de problemas intercomunales e intracomunales, y en la mira de miembros de comunidades aledañas, empresarios y productores madereros que habían pretendido la explotación ilegal, la compra de terrenos o su alquiler fuera de la ley. Si bien la confrontación por el aprovechamiento de los recursos forestales había sido un hecho "cotidiano" para los miembros de esta comunidad, la situación se tornó insostenible en el año 2008, cuando talamontes vinculados al crimen organizado se constituyeron como los principales agentes que dominaron el usufructo del bosque. Estos "nuevos" actores sociales no solo se enfocaron en la explotación de los recursos forestales sino que también generaron un ambiente de violencia a través de acciones como el incendio de pastizales, extorsiones, amenazas, secuestros e incluso el asesinato de algunos comuneros.

La tala ilegal en San Francisco de Cherán puede interpretarse como el agravio que dio lugar al movimiento; no obstante, la referencia empírica apunta más a un cambio en el control y la distribución de los recursos forestales -y del territorio-, en un lapso de tiempo que va del surgimiento de los primeros grupos de talamontes ilegales hasta su asociación con el crimen organizado (2007-2011). Estos buscaron incorporar otras formas de aprovechamiento del territorio a través de la violencia. El deterioro de la cobertura forestal ya era visible antes de las acciones ilegales (2008) y se relacionaba con las necesidades crecientes de alimentación, con la búsqueda de los medios de trabajo e incluso con la tala ilegal a pequeña escala. No obstante, la mayor deforestación se produjo entre los años 2010 y 2011 (España y Champo, 2016), debido al saqueo orquestado por grupos de talamontes, que, vinculados al crimen organizado, deseaban el control de las tierras para la explotación ilegal de madera e incluso para establecer cultivos de narcóticos.

Dichas acciones fueron legitimadas por la corrupción de las autoridades tanto municipales como estatales, las cuales se aliaron con los oponentes al movimiento e ignoraron hechos como el asesinato y la desaparición forzada de algunos integrantes de la comunidad. A lo anterior, se sumaron las divisiones entre los chenarenses simpatizantes del Partido Revolucionario Institucional (PRI) y los del Partido de la Revolución Democrática (PRD), mismas que se profundizaron durante el último proceso electoral en el municipio (2007).

La acción colectiva para frenar el saqueo del bosque se inició el día 14 de abril de 2011 mediante una discreta convocatoria que invitaba a la población a poner alto a esta serie de agravios. De acuerdo con la información recolectada durante el trabajo de campo fueron las mujeres quienes durante la madrugada del 
15 de abril decidieron emprender una acción colectiva, no solo por la defensa de los recursos del bosque sino también por la conservación de las fuentes de agua natural, que comenzaban a ser afectadas debido a los incendios y a la tala inmoderada provocada por los talamontes.

En esta primera fase del movimiento -a la que denominaremos fase de visibilidad-, la gente de la comunidad movilizó los recursos materiales más simples, como palos, herramientas de trabajo, pirotecnia, y todo aquello que sirviera de arma para entablar una confrontación abierta con los talamontes y con los grupos que les brindaban protección. Las redes de solidaridad y apoyo mutuo fungieron como canales de movilización de este y otro tipo de recursos (intangibles), tales como el conocimiento, el apego al territorio y los acuerdos tomados entre la gente de la comunidad durante reuniones previas a esa madrugada. Estas formas de apoyo pueden ser consideradas como recursos funcionales a la acción colectiva, pero también como estructuras de movilización en sí mismas que, si bien responden a patrones espontáneos y aislados de organización, pueden ser vistas como redes comunitarias informales que subyacían a las formas de temor implementadas por los talamontes y sus aliados -el crimen organizado y la policía municipal y estatal-, y bajo el aparente escenario de divisionismo propiciado por dos de las principales fuerzas políticas en el país.

La madrugada de la confrontación constituyó una oportunidad política inicial para hacer resurgir esas estructuras organizativas locales que -basadas en el parentesco, territorialidad, actividades productivas, identidad colectiva- promovieron la circulación de los recursos (materiales e inmateriales) esenciales para esta primera acción. Esta sería solo una muestra del amplio repertorio de acciones colectivas que se suscitarían a mediano y largo plazo durante el MS. Los canales y los recursos movilizados durante la fase de visibilidad, no rebasaron el ámbito de lo comunitario. Los enfrentamientos que la comunidad de Cherán había mantenido con las comunidades colindantes por cuestiones de fijación de límites, invasión de pastos o bosques, venta o explotación ilegal de tierras, entre otros, no dieron cabida a que las estructuras organizativas comunitarias se apoyaran en recursos asociativos extracomunitarios formales e informales.

La autorganización como recurso y estructura de este MS partió de los marcos organizativos que han regido la vida comunitaria de Cherán, los cuales se fundamentan en principios como la no existencia de un centro jerárquico y en interacciones más o menos sostenidas entre los pobladores a través del intercambio de recursos, de la negociación de prioridades y la toma de decisiones en los proyectos públicos. Para los habitantes de Cherán, la comunidad ha sido la forma colectiva más importante de organización y la asamblea de comuneros el órgano principal de gestión. El origen étnico de esta comunidad también otorga un sentido colectivo a sus formas de organización, que se caracterizan por ser descentralizadas y predominantemente democráticas pero ante todo reforzadas por los lazos interpersonales de solidaridad y compromiso histórico de los chenarenses hacia su territorio.

Las oportunidades políticas también son esencia y motor de la acción colectiva. Desde una visión microsociológica -y para esta fase del MS - se identificaron diversos sucesos que, a manera de oportunidades políticas, constituyeron los principales alicientes entre los pobladores locales. El principal incentivo a nivel colectivo fue detener la devastación de las fuentes de vida de la comunidad (bosque y agua). A este se sumaron otros de orden particular, tales como la lucha por un ambiente de tranquilidad en la comunidad, debido a los actos de violencia en contra de quienes se opusieran al despojo de los recursos naturales.

En términos macrosociales, la debilidad institucional constituyó una oportunidad política para retornar a las formas de organización comunitaria. Mientras se libraban algunas luchas internas entre grupos del partido político (PRD) que había gobernado el municipio de Cherán desde 1989 a 2007 y el partido dominante en el país (PRI), la población chenarense tomaba la presidencia municipal de Cherán, la cual encontraba en sus propios recursos y estructuras organizativas comunitarias la fuerza para hacer frente a la apropiación violenta de sus recursos desplegada por los adversarios. 


\subsection{El desarrollo del movimiento: la organización formal y las redes de apoyo}

La fase del desarrollo del movimiento se concibe como el proceso organizado conformado por la movilización de los recursos necesarios y por el aprovechamiento de las oportunidades políticas para tratar establecer el cambio social. Durante esta fase se identificaron dos recursos que lograron incidir tanto en la recuperación del control del territorio como en el reconocimiento legal de su derecho a la autodeterminación: 1) la movilización social y política a través de diversas formas y niveles de organización, y 2) la movilización del derecho.

Después de la primera confrontación abierta entre la comunidad y los talamontes, la cohesión comunal y la capacidad de organización de la población fueron puestas a prueba. Lo que avivó la organización comunitaria durante la madrugada del 15 de abril de 2011 fue el temor a que los integrantes del crimen organizado regresaran a causar desgracia y desolación, pues su capacidad para generar violencia estaba demostrada con hechos pasados.

De acuerdo con Tilly (1978), el potencial de un movimiento está determinado por la presencia de grupos con identidades fuertes y redes interpersonales densas exclusivas de los miembros del movimiento. En este mismo sentido, Tarrow (2004) afirma que los movimientos sociales encuentran sus raíces en redes sociales preexistentes, que son depositarias de relaciones de confianza, reciprocidad y aprendizaje cultural. En el caso de Cherán, el desarrollo del movimiento no solo se alimentó de los recursos y de la fortaleza de las redes internas, sino también de las contribuciones y las redes extralocales que en conjunto aprovecharon las oportunidades políticas para fortalecer esta acción colectiva.

Las organizaciones comunitarias del movimiento social (OCMS) se caracterizaron en su mayoría por ser estructuras locales, de naturaleza informal, no jerárquicas, participativas y democráticas. Para fines de este trabajo se distinguen las OCMS de resistencia y protección, y las de consenso. Las primeras se identifican como organizaciones espontáneas, sin estructura interna, con integrantes sin roles definidos y con fines de protección del territorio, aunque con el paso del tiempo se fueron constituyendo como estructuras formalizadas de consenso. A diferencia de estas, las de consenso se caracterizaron por ser menos flexibles, pero no por ello dejaron de lado el modelo democrático y participativo, que maximizó el compromiso comunitario en la toma de decisiones respecto de las acciones a favor del movimiento.

\subsubsection{Organizaciones comunitarias del movimiento social (OCMS): la resistencia y protección del territorio}

Las estructuras organizativas para la resistencia y protección adoptaron la forma de redes territoriales. Las barricadas, las fogatas y la ronda comunitaria fueron los primeros canales formales de movilización que se desplegaron en las salidas y en cada una de las esquinas de los cuatro barrios que conforman el municipio después de la confrontación que la comunidad entabló con los talamontes. Estas formas de organización se integraron como organizaciones de protección del territorio y de la propia población ante el temor de que los oponentes regresaran a tomar venganza.

Particularmente, las fogatas se convirtieron en espacios para las prácticas cotidianas, la transmisión de información y, posteriormente, para la toma de decisiones en torno al movimiento. Los pobladores de los diferentes barrios se encargaron de su acondicionamiento con retazos de madera, láminas y otros materiales que ayudaron a soportar las condiciones climáticas durante los días en que se mantuvo activo el movimiento en las calles del municipio. Las mujeres dispusieron de lo necesario para proveer de alimentos a quienes participaban de las actividades, y a los niños y niñas se les impartían ahí mismo las clases y otras enseñanzas.

Los recursos intangibles fueron una constante en el funcionamiento de estas estructuras organizativas: los lazos de solidaridad, el conocimiento sobre la comunidad e incluso las habilidades para la resolución de 
problemas fueron puestos en juego para discutir, analizar y posteriormente tomar decisiones respecto a los asuntos del movimiento. Si bien inicialmente las fogatas no tuvieron una estructura formal interna (roles, reglas, normas, procesos), con el desarrollo de la acción colectiva surgió la figura del representante de fogata, que intervenía como interlocutor entre los integrantes de otras fogatas y las organizaciones comunitarias de consenso (OCC), tales como la Asamblea General de Comuneros (AGC) o la Coordinación General del Movimiento de Lucha (CGML). Con ello las fogatas se convirtieron en una estructura de consenso.

La ronda comunitaria también formó parte de este tipo de estructuras. Esta es una forma antigua de policía en las comunidades rurales que deriva de la organización comunal para procurar la seguridad y defensa de los recursos comunitarios. Casi a la par de la formación de la Coordinación General del Movimiento de Lucha (2011), la ronda comunitaria resurgió y llegó a constituirse por 95 habitantes de la comunidad. Si bien inicialmente no contaba con recursos para su funcionamiento (transportes, uniformes, armas), sus integrantes encontraron en la confrontación con el gobierno municipal la oportunidad para apropiarse de patrullas y armas, a fin de realizar los recorridos de vigilancia hacia las zonas más vulnerables del territorio.

El MS en Cherán partió de estructuras organizativas preexistentes que constituyeron estructuras de movilización de origen histórico, las cuales fueron reivindicadas durante el movimiento a partir de recursos comunitarios e institucionales. Estas organizaciones comunitarias aprovecharon la cooptación de los cuerpos policiacos por parte del crimen organizado como una oportunidad para hacer frente de manera colectiva a los oponentes. Además, sirvieron como estrategia inmediata para recuperar en un mediano plazo el control del territorio. Los MS no solo encuentran su fuerza en las organizaciones formales macro sino también en estructuras de cualquier nivel de organización (Ramírez, 2016).

\subsubsection{Organizaciones comunitarias de consenso y las redes extralocales de movilización}

En el caso de Cherán, las estructuras de resistencia y protección de la comunidad representaron ese primer momento de movilización de recursos, de organización y de búsqueda de oportunidades políticas a nivel micro/meso. Particularmente, las estructuras de consenso fueron espacios para la movilización de recursos y la construcción de estrategias/alianzas para la búsqueda de oportunidades políticas a nivel estatal, nacional e incluso internacional.

Las estructuras de consenso conformadas en el desarrollo del MS de Cherán tuvieron como punto de partida la organización comunal, por tanto, reprodujeron las formas de organización cotidiana, familiar y comunitaria. La Asamblea General de Comuneros (AGC) es una organización que históricamente ha regido los asuntos de la vida comunitaria y de la tenencia de la tierra en Cherán.

La fortaleza y el protagonismo adquirido por la AGC a raíz de la inestabilidad del gobierno municipal generó las condiciones para que sus integrantes comenzaran a definir la estructura (sector organizado) del MS. Sin más recursos que la credibilidad y solidaridad de la mayoría de los comuneros y sus familias, una de las primeras acciones fue la conformación de la Coordinación General del Movimiento de Lucha (CGML). Este canal de movilización fue integrado por cuatro comuneros -electos en asambleas-, uno por cada uno de los barrios que conforman el municipio, quienes en conjunto no solo se encargaron de encabezar la organización, sino también el proceso judicial que la comunidad emprendería posteriormente.

A lo largo del desarrollo del movimiento, la comunicación y la toma de decisiones a nivel interno fluyó en dos direcciones de la AGC y de la CGML: hacia los miembros de las fogatas, las barricadas y la ronda comunitaria, y viceversa. En este sentido, los recursos para el movimiento (información, recursos en especie, apoyo comunitario) se mantuvieron disponibles para las acciones necesarias.

En ausencia del gobierno municipal, las necesidades cotidianas de la comunidad quedaron a la deriva. La CGM se involucró en la conformación de doce comisiones (justicia, víveres, salud, educación, cultura, prensa y propaganda, forestal, agrícola, ganadería, finanzas, agua, jóvenes y de identidad) que velaron por el buen funcionamiento de los asuntos de la comunidad y que, de alguna forma, fueron también el soporte de las 
necesidades del propio movimiento; la estructura de organización comunitaria se puso al servicio de la acción colectiva.

A diferencia de otros movimientos contemporáneos, la preexistencia de ambas estructuras de organización permite ubicar esta acción colectiva en Cherán dentro de la concepción clásica de los movimientos que se caracterizan por la presencia de liderazgos autóctonos, la filiación de personal voluntario, lo que da lugar a una estructura construida "desde abajo", con recursos de los beneficiarios directos y acciones basadas en la participación masiva.

El objetivo inicial del movimiento era recuperar las zonas de bosque que estaban siendo devastadas por la tala ilegal. No obstante, de la conservación de este recurso natural dependía que la comunidad mantuviera el control del territorio más allá del movimiento. En este sentido, la CGML con el apoyo de las redes extralocales encontró la vía para salvaguardar los recursos en la legitimidad institucional de la estructura organizativa del MS. Dicha legitimidad contribuyó a orientar la lucha hacia el reconocimiento legal a la libre determinación de este pueblo como una forma de derecho que no solo le permitiría elegir su devenir en términos territoriales, sino también en relación a la vida económica, política y cultural de la comunidad.

En este proceso, el apoyo de las redes extralocales fue fundamental para lograr la visibilidad y el posicionamiento del MS de Cherán en la agenda de las Organizaciones no Gubernamentales (ONGs) internacionales, con la finalidad de que estas últimas contribuyeran a ejercer presión sobre las instituciones del estado. Cabe destacar que las redes de apoyo no solo buscaron posicionar el conflicto como un asunto de índole ambiental, sino también como una lucha en defensa de la autonomía de este pueblo indígena.

Los actores individuales y colectivos que conformaron las redes extralocales de apoyo se caracterizaron por integrar un complejo entramado de alianzas simbólicas, solidarias y estratégicas, que permitieron la movilización de diferentes recursos y, al mismo tiempo, la exploración de vías alternativas (jurídica, presión política, opinión pública) para alcanzar el "nuevo" objetivo del MS. A nivel regional, estatal y nacional destacaron las organizaciones de base comunitaria (OBC), los sindicatos, colectivos sociales de orden nacional e internacional, e incluso grupos de abogados activistas, intelectuales y artistas (músicos, actores, escritores) (ver Figura 3).

La sinergia entre estos actores sociales se generó principalmente por su identificación político-ideológica con el movimiento y su adhesión permitió conformar un sujeto político de apoyo (estructura organizativa) con capacidad para movilizar recursos estratégicos tales como la opinión pública en medios de comunicación, las redes virtuales, la experiencia de cada una de las organizaciones en la lucha por diversos recursos ante organismos internacionales, las redes entabladas con otras organizaciones y las alianzas políticas.

Por otra parte, algunas de las organizaciones nacionales de apoyo al MS experimentaron episodios de intervención estatal en el interior de sus estructuras. El apoyo otorgado al MS de Cherán se manejó como un conglomerado heterogéneo con fuertes alianzas a nivel internacional, pero con pocas posibilidades de promover el patrocinio de algunos actores políticos a escala nacional para el movimiento (tales como los partidos). 


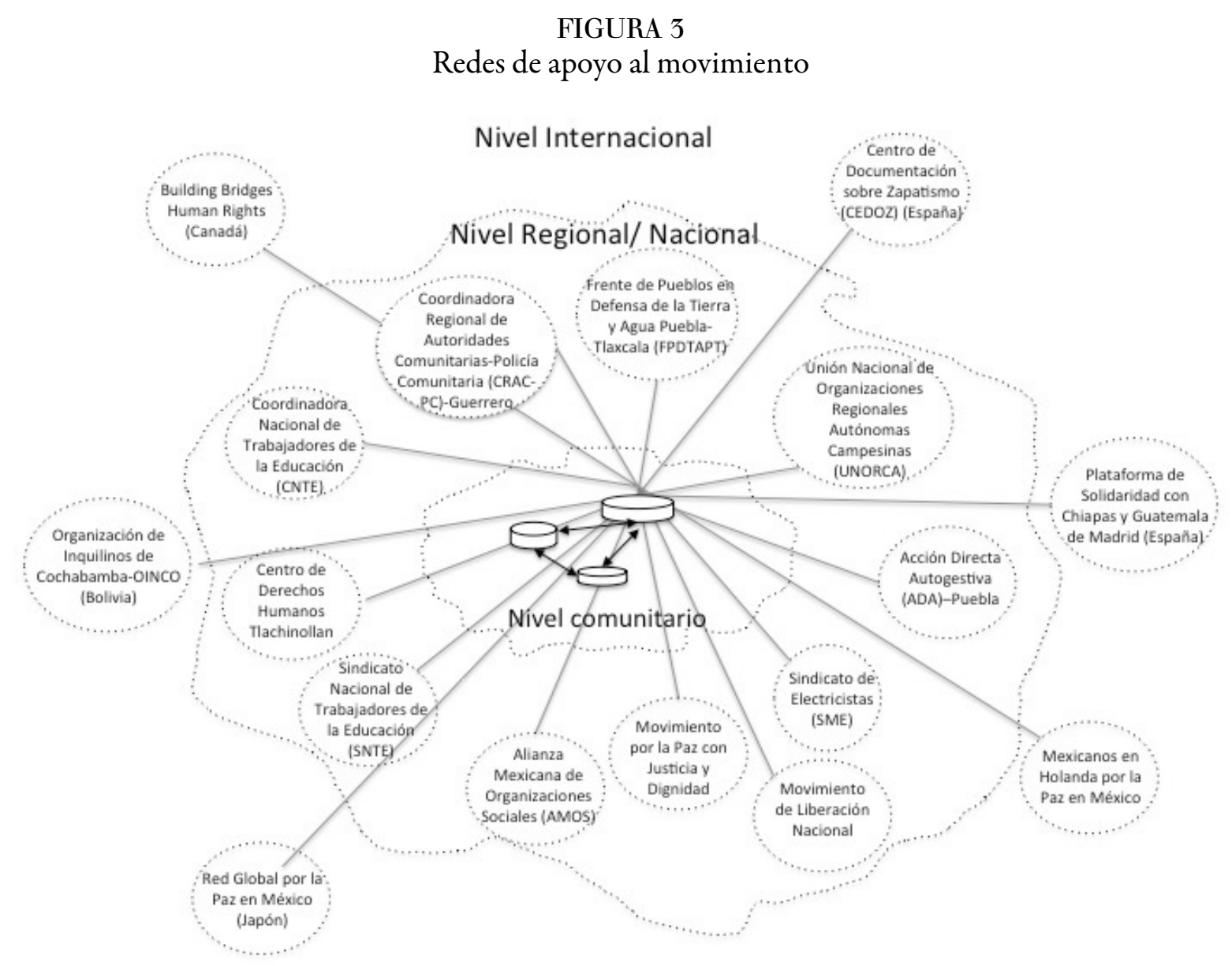

Elaboración propia a partir del trabajo de campo

Las estructuras extra locales de movilización que conformaron este MS habrían tenido mayor impacto en la esfera de las decisiones políticas, si las oportunidades de índole coyuntural (la disponibilidad de aliados, los alineamientos electorales y la cohesión de élite) no hubieran limitado la alianzas entre las estructuras del movimiento y los actores políticos.

Por un lado, se observó la ruptura entre los grupos organizados a nivel comunitario y el gobierno (a nivel estatal y federal), debido a la debilidad con que las instancias gubernamentales respondieron a la demanda de protección hecha por la comunidad de Cherán. Por el otro, se identificó que el proceso electoral para elegir gobernador, diputados y presidentes municipales, que se desarrolló poco después del inicio del movimiento (noviembre 2011), no fue más que otro intento de clientelismo electoral por parte de los partidos políticos para asegurar la realización de las elecciones en la comunidad. De hecho, las omisiones y las actitudes oportunistas del gobierno antes y durante el desarrollo del movimiento condujo a los chenarenses -a través de un acuerdo de asamblea celebrado el 1 de junio de 2011- a tomar la decisión de no participar ni dejar instalar las casillas para que se llevaran a cabo las elecciones en la comunidad.

En resumen, se puede afirmar que ni las alianzas políticas, ni los posibles alineamientos electorales ni mucho menos el proceso electoral constituyeron oportunidades políticas para que tanto las estructuras de consenso del movimiento como las redes extralocales pudieran establecer canales de acceso que les permitieran colocar las demandas del movimiento en la agenda política del país. Era de suponer que el ambiente electoral haría que los partidos políticos a nivel municipal y estatal prestaran atención a esta comunidad, debido a la posibilidad 
de que pudieran ser afectados los resultados electorales; sin embargo, esto no sucedió. A pesar de dicho escenario, la idea de la autonomía y la libre determinación de este pueblo indígena tomó cada vez más fuerza.

\subsection{La movilización del derecho}

Si bien el movimiento estuvo apoyado por una amplia gama de actores colectivos, que funcionaron como una estructura de movilización al exterior, en este apartado se pretende enfatizar la movilización del derecho que, con la orientación de algunos abogados activistas, permitió a las organizaciones de consenso del MS emprender un activismo jurídico para que la comunidad ejerciera su derecho a controlar sus recursos naturales y establecer sus propios sistemas normativos en las distintas esferas de la vida comunitaria.

El derecho como instrumento pero también como oportunidad política, les permitió a las estructuras organizativas del movimiento encontrar en el sistema legal - derechos reconocidos en tratados internaciones y en el ordenamiento jurídico nacional- los puntos de acceso o los canales institucionales disponibles para incidir en las decisiones políticas. Alcanzar el reconocimiento legal de su derecho a elegir sus autoridades a través de "usos y costumbres" fue el objetivo de esta faceta en el desarrollo del MS. La aparente descentralización del poder en el gobierno mexicano fue un factor que dio cabida a encontrar en las diversas instancias (poder ejecutivo, legislativo y judicial) las oportunidades políticas para alcanzar dicho objetivo. De acuerdo con Favela y Murillo (2002), un sistema en el que las decisiones son tomadas por el poder ejecutivo tiene menos puntos de acceso a dicha toma de decisiones. Por tanto, mientras más descentralizada es la distribución del poder, mayores son las oportunidades de que la movilización popular se desarrolle y tenga éxito. En este contexto, las legislaturas y los tribunales pueden constituir puntos de acceso para el proceso de toma de decisiones.

Una vez canceladas las elecciones municipales y, aprovechando la aparente distribución de competencias entre los diferentes niveles del gobierno, los representantes del MS solicitaron -el 26 de agosto de $2011-$ al Instituto Electoral del estado de Michoacán poder llevar a cabo la elección de sus autoridades municipales a través del sistema de "usos y costumbres". Este hecho constituyó un primer intento de poner en juego los recursos legales a través del grupo de abogados activistas. El Instituto se declaró no competente para resolver tal petición.

A raíz de la resolución, las estructuras de consenso del movimiento con el respaldo de algunos integrantes de las redes de apoyo, decidieron ampliar la movilización de recursos políticos mediante la puesta en marcha de un juicio para la protección de los derechos políticos del ciudadano. La importancia del asunto fue tal que dicha causa fue retomada por la Sala Superior del Tribunal Electoral del Poder Judicial de la Federación (Trife). El 2 de noviembre de 2011, apoyado en la movilización del derecho internacional, el Trife determinó que la comunidad de Cherán tenía derecho a solicitar la elección de sus autoridades de acuerdo con sus procedimientos tradicionales, y dejó fuera toda posibilidad de la organización de elecciones bajo el régimen del sistema de partidos.

La ruptura de las organizaciones comunitarias con las autoridades tanto municipales como estatales y con los partidos políticos no le impidió al movimiento encontrar en el escenario de las oportunidades políticas "aliados" como el Trife, el cual coaccionado por el derecho internacional, tuvo que reconocer la autonomía y el derecho a la libre determinación a este pueblo indígena. Lo anterior no solo permitió la movilización del derecho a favor del movimiento, sino que también dio lugar a la activación de otro tipo de recursos, tales como la atención de los medios de comunicación. La visibilidad mediática fue indispensable para presionar a las autoridades a dar cumplimiento a la determinación tomada por el tribunal.

Cabe destacar que, en esta faceta, el aprovechamiento de las oportunidades coyunturales y estructurales brindadas por el entorno político a favor del movimiento permitió la apertura de diferentes canales institucionales a través de los cuales fueron manifestadas las demandas del MS. Sin embargo, hubo otras 
condiciones que no necesariamente lo beneficiaron, tales como el menoscabo de la protesta a través de los medios de comunicación y la cooptación de algunos integrantes clave, entre otras acciones.

\section{Discusión}

En este trabajo se pone de manifiesto la dinámica interna de la acción colectiva en defensa de los recursos forestales. El abordaje teórico también permite la discusión de aspectos como el origen de los MS, los movimientos como actores racionales y organizados, y como entidades que interactúan con otras organizaciones y aprovechan las condiciones del entorno para propiciar el cambio social.

Los principales paradigmas en torno a los MS -la teoría de la sociedad de masas, la privación relativa, la teoría del comportamiento colectivo (Gurr, 1970; De la Garza, 2011) - apuntan a que los aumentos en los reclamos individuales generados por cambios sociales o situaciones desventajosas (Gusfield, 1982, 1994) pueden dar origen a la formación de un MS. En el caso de Cherán, concentrarse en la serie de agravios auspiciados por los adversarios es suficiente para explicar que el origen de este MS no está en los cambios en las relaciones de poder (Korpi, 1974) ni en los conflictos de intereses estructurales. Aunque los agravios contribuyeron a su formación, los factores determinantes fueron, por un lado, el creciente deterioro de la calidad y cantidad de los recursos bosque y agua y el cambio en el control y aprovechamiento de los recursos forestales y, por el otro, la disponibilidad de organizaciones formales e informales capaces de movilizar la insatisfacción individual y los conflictos sociales en contra del orden establecido (Riechman y Fernández, 1995). En este caso, esto ocurrió por los oponentes y autoridades municipales y estales en relación a la depredación del bosque.

Las organizaciones como elementos medulares en la visibilidad, desarrollo y continuidad del movimiento en Cherán encontraron su potencial de movilización en las formas de organización preexistentes a nivel local (Craig, 1983). Si bien otros autores han incorporado el paradigma de la identidad colectiva como motor de los MS (Melucci, 1995, 1996; Touraine, 1995; Chihu y López, 2007), este trabajo es un análisis estructural, más que interpretativo de la subjetividad de los actores. No obstante, se reconoce en el trabajo empírico que las solidaridades y los compromisos morales producto de la identidad indígena y campesina de este pueblo funcionaron a manera de incentivos colectivos determinantes en la reivindicación de las organizaciones comunitarias. De manera general, no debe ignorarse que la mayoría de los movimientos están conformados por sectores organizados y estructurados, y por otras partes más fluidas (Tarrow, 1997; Raschke, 1994). Esto tiene que ver con el grado de organización alcanzado por un movimiento, pero en el caso de Cherán se observó una dicotomía interesante entre la organización y la formalización en los canales de movilización; la primera, representada por el conjunto de organizaciones formales e informales del movimiento y, la segunda, comprendida por las normas y los procesos intencionados que regularon el comportamiento de esas organizaciones, tales como la toma de decisiones, la diferenciación de tareas y funciones, entre otras. Lo anterior confirió al movimiento un equilibrio dinámico entre lo formal y lo informal, y la eficiencia de movilizar los recursos y las oportunidades del entorno a favor de la consecución del cambio social buscado por este movimiento.

La teoría de la organización tradicional de los MS es cada vez más inapropiada (Craig, 1994; Tarrow, 2002), debido a que no contempla que la nueva dinámica organizativa de los movimientos incluye un segundo nivel de organización: las redes de apoyo. Si bien las organizaciones y colectivos de apoyo son parte de la infraestructura utilizada por el movimiento de Cherán para alcanzar sus objetivos, también actuaron como sujetos sociopolíticos cuyas acciones más importantes fueron la visibilidad política del movimiento y la movilización del derecho. 


\section{Conclusiones}

La emergencia de movimientos como el suscitado en Cherán es ejemplo de la demanda de los derechos colectivos de los pueblos indígenas. Más allá de lo particular que resulta este caso, su estudio da luz sobre las características que distinguen a la mayoría de los movimientos populares -tanto por sus características organizativas como por sus elementos identitarios y conceptualizaciones de lo colectivo- que se colocan en el escenario de la disputa por los recursos naturales y los territorios en México.

Considerar la identidad como elemento que configura la dinámica interna de los MS es una de las limitantes que presenta el análisis de los MS desde la TMR. En el estudio del movimiento de Cherán se evidencia -sin abordarlo ampliamente- que la identidad se construye en la estructura del conflicto; por tanto, es también un elemento integrador, pues no es posible pensar en un movimiento "vacío", es decir, sin ideales ni principios que guíen las acciones. En cuanto a los alcances, esta postura puede ser vinculada al enfoque de redes (network approach), del cual pueden derivar análisis de redes sociales que evidencien el potencial de movilización a nivel socioespacial de algunas comunidades, sobre todo en movimientos vinculados a la conservación, los cuales en las últimas décadas han adquirido importancia a escala internacional.

\section{REFERENCIAS}

Aguilar, A., Lafosse, S., Rojas, H., \& Steward, R. (2010). The Constitutional Recognition of Indigenous Peoples in Latin America. International Law Review, 2(2), 44-96. Recuperado de https://core.ac.uk/download/pdf/4671 0889.pdf

Aragón, O. (2013). El derecho en insurrección. El uso contra-hegemónico del derecho en el movimiento purépecha de Cherán. Revista de Estudios \& Pesquisas sobre as Américas, 7(2), 37-69. Recuperado de: https://www.researchgate.net/publication/316901217 EL DERECHO EN INSURRECCION El us o contra-hegemonico_del derecho_en_el movimiento purepecha de cheran

Aragón, O. (2016). ¿Por qué pensar desde las epistemologías del sur la experiencia política de Cherán? Un alegato por la igualdad e interculturalidad radical en México. Nueva Antropología, 29(84), 143-161.

Berrío, A. (2006). La perspectiva de los nuevos movimientos sociales en las obras de Sydney Tarrow, Alain Touraine y Alberto Melucci. Estudios Politicos, 29, 219-236. Recuperado de http://www.redalyc.org/articulo.oa?id=164 29057009

Brunet, I. y Pizzi, A. (2010). La Acción Colectiva desde la Teoría de la Movilización de Recursos. Sociedady Utopia. Revista de Ciencias Sociales, 36, 27- 38. Recuperado de https://dialnet.unirioja.es/servlet/articulo?codigo=342 9865

Calveiro, P. (2014). Repensar y ampliar la democracia. El caso del Municipio Autónomo de Cherán K'eri. Argumentos, $27(75), 193-212$.

Chihu, A. y López, A. (2007). La construcción de la identidad colectiva en Alberto Melucci. Polis, 3(1), 125-159. Recuperado de https://polismexico.izt.uam.mx/index.php/rp/article/view/318

Composto, C. (2012). Acumulación por despojo y neoextractivismo en América Latina. Una reflexión crítica acerca del Estado y los movimientos socio-ambientales en el nuevo siglo. Astrolabio, 8, 323-352. Recuperado de https: //revistas.unc.edu.ar/index.php/astrolabio/article/view/767/1031

Craig, J. (1983). Resource mobilization Theory and the Study of Social Movements. Annual Review of Sociology, 9 , 527-553. Recuperado de https://www.jstor.org/stable/2946077

Craig, J. (1994). La teoría de la mobilización de recursos y el estudio de los movimientos sociales. Zona Abierta, 69, 5-49. Recuperado de https://www.ses.unam.mx/docencia/2015II/Jenkins1994_LaTeoriaDeLaMovilizacionD eRecursos.pdf 
Eika Cruz Coria, et al. La defensa de los recursos forestales: el movimiento social en La ComuniD...

De la Garza, R. (2011). Las teorías de los movimientos sociales y el enfoque multidimensional. Estudios politicos, 22, 107-148. Recuperado de http://www.scielo.org.mx/scielo.php?script=sci_arttext\&pid=S0185-161620110001 00007

España, M. L., y Champo, O. (2016). Proceso de deforestación en el municipio de Cherán Michoacan, México (2006-2012). Madera y Bosques, 22(1), 141-153. Recuperado de http://www.scielo.org.mx/scielo.php?script= sci_arttext\&pid=S1405-04712016000100141

Favela, D., y Murillo, L. (2002). La estructura de oportunidades políticas de los movimientos sociales en sistemas políticos cerrados: examen del caso mexicano. Estudios Sociológicos, 20 (58), 91-121. Recuperado de http://ww w.redalyc.org/articulo.oa?id $=59805805$

Fernández, J. M.. y Antolín, J. E. (2000). Estructura organizativa de los "nuevos" movimientos sociales en el País Vasco: claves para su comprensión. Politica y Sociedad, 35, 165-173. Recuperado de https://revistas.ucm.es/index.php /POSO/article/view/POSO0000330153A

Ferree, M. (1994). El contexto político de la racionalidad: las teorías de la elección racional y la movilización de los recursos. En E. Laraña y J. Gusfield (Eds.), Los nuevos movimientos sociales. De la ideología a la identida, 151-182. Madrid: CIS.

Forlani, N. (2014). Territorialidades, ciudades y agronegocio. Fundamentos en Humanidades, XV(29), 223-249. Recuperado de http://www.redalyc.org/pdf/184/Resumenes/Resumen_18447748009_1.pdf

Galafassi, G. (2011). Teorías diversas en el estudio de los movimientos sociales. Una aproximación a partir del análisis de sus categorías fundamentales. Movimientos Sociales, 6(11), 7-32. Recuperado de http://www.scielo.org.mx/s cielo.php?script=sci_arttext\&pid=S2007-81102011000200001

Gamson, W. (1975). The Strategy of Social Protest. Homewood II.: Dosey.

Garretón, M. (2002). La transformación de la acción colectiva en América Latina. Revista de la CEPAL, 76, 7-24. Recuperado de https://repositorio.cepal.org/bitstream/handle/11362/10797/1/076007024_es.pdf

González, A., y Zertuche, V. (2017). Cherán: Cinco años de un gobierno autónomo (2017). En G. Pleyers y M. Garza (Coord.), México en movimientos: resistencias y alternativas, 29-35. México: Universidad Autónoma Benito Juárez de Oaxaca/Universidad de Ciudad Juárez/Miguel Ángel Porrúa.

Gurr, T. (1970). Why Men Rebel. Princeton University Press: Princeton.

Gusfield, J. (1982). Social Movements and Social Change: Perspectives of Linearity and Fluidity. En L. Kriesberg (Ed.), Research in Social Movements, Conflict and Change, 47-63. JAI Press: Greenwich, CT.

Gusfield, J. (1994). The Reflexivity of Social Movements: Collective Behaviour and Mass Society Theory Revisited. En E. Laran, H. Johnston \& J. Gusfield (Eds.), New Social Movements: From Ideology to Identity, 78-92. Temple University Press: Philadelphia.

Instituto Nacional de Estadística y Geografía (30 de Noviembre de 2007). Instituto Nacional de Estadística y Geografía. Recuperado el 3 de Agosto de 2018, de Censos Agropecuarios. Censo Agrícola, Ganadero y Forestal 2007: https://www3.inegi.org.mx/sistemas/tabuladosbasicos/default.aspx?c=17177\&s=est

Korpi, W. (1974). Conflict, power and relative deprivation. American Political Science Review, 68(4), 1569-1578. Recuperado de https://www.jstor.org/stable/1959942

Lang, M. (2012). Crisis civilizatorias y desafíos para las izquierdas. En M. Lang y D. Mokrani (Coomp.), Más allá del desarrollo, 7-18. México: Fundación Rosa Luxemburgo/ Abya Yala.

Laraña, E. (1999). La construcción de los movimientos sociales. Madrid: Alianza.

León, A. (2015). Los nuevos movimientos sociales de México en el siglo XXI. Estudio exploratorio. Espacios transnacionales, 2(4), 14-25. Recuperado de http://espaciostransnacionales.org/wp-content/uploads/2015/07 /ET4-LEON-.pdf

Mandujano, M. (2014). Autodeterminación y liberación en el marco de la reinvención del Estado Moderno. Memorias del VI Simposio Becarios CONACYT. Estrasburgo: Universidad de La Laguna, pp.1-8.

March, J., \& Olsen, J. (1989). Rediscovering Institutions: The Organizational Basis of Politics. New York: Free Press. 
Marín, R., y Ramírez, S. (2014). Movimientos no convencionales para la recosntrucción de las instituciones politicas en Michoacán: el caso de Cherán. Trabajo presentado en el Segundo Congreso Internacional de la Asociación Mexicana de Ciencia Política, Toluca, Estado de México.

Martí, S. (2004). Los movimientos sociales en el mundo globalizado ¿Alguna novedad?. América Latina Hoy, 36, 79-100. Recuperado de http://revistas.usal.es/index.php/1130-2887/article/view/7413

Martínez, J. (2017). San Francisco Cherán. Revuelta comunitaria por la autonomía, la reapropiación territorial y la identidad. Economia y sociedad, XXI(36), 145-166. Recuperado de https://www.redalyc.org/pdf/510/510520 64009.pdf

Mc Adam, D., Mc Carthy, J., y Zald, M. (1999). Movimientos sociales: Perspectivas comparadas. Oportunidades Politicas, Estructuras de Movilización y Marcos Interpretativos Culturales. España: Ediciones Istmo.

McCarthy, J. y Zald, M. (1977). Resource Mobilization and Social Movements: A Parcial Theory. The American Journal of Sociology, 82(6), 1212-1241. Recuperado de https://www.jstor.org/stable/2777934

Melucci, A. (1995). The Process of Collective Identity. En H. Johnston y B. Klandermans (Eds.), Social Movements and Culture, 41-63. Minneapolis: University of Minnesota Press.

Oberschall, A. (1973). Social Conflict and Social Movements. New Jersey, EEUU: Prentice-Hall.

Ojeda, L. (2015). Cherán: el poder del consenso y las políticas comunitarias. Política Común, 7, 1-7. Recuperado de: en: https://quod.lib.umich.edu/p/pc/12322227.0007.007?view=text;rgn=main

Ortíz, M., y Madrid, S. (2017). Territorios forestales comunitarios amenazados por la minería en México. México: Consejo Civil Mexicano para la Silvicultura Sustentable, A.C.

Pizzi, A., y Brunet, I. (2012). Nuevas formas del movimiento obrero: las empresas recuperadas por sus trabajadores en Argentina. Articulaciones políticas y estrategias de inserción. Cuadernos de Relaciones Laborales, 30(2), 563-583. Recuperado de https://revistas.ucm.es/index.php/CRLA/article/view/40213

Puricelli, S. (2005). La Teoría de Movilización de Recursos desnuda en América Latina. Revista Theomai, (12). Recuperado de http://www.redalyc.org/pdf/124/12401202.pdf

Ramírez, M. A. (2016). A manera de introducción. Los movimientos sociales en los albores del siglo XXI. En M. Ramírez (Coord.), Movimientos sociales en México: apuntes teóricos y estudios de caso, 19-57. México: Universidad Autónoma Metropolitana.

Raschke, K. (1994). Sobre el concepto de movimiento social, Zona Abierta, 69, 121-152. Recuperado de https://ww w.ses.unam.mx/docencia/2014II/Raschke1994_SobreElConceptoDeMovimientoSocial.pdf

Riechman, J., y Fernández, F. (1995). Redes que dan libertad. Introducción a los nuevos movimientos sociales. Barcelona: Paidós.

Santillán, V. (2014). El ejercicio del poder desde la resistencia indígena. Cherán, Michoacán 2011-2014 (Tesis de Maestría). Facultad Latinoamericana de Ciencias Sociales, Ciudad de México. México

Secretaría de Medio Ambiente y Recursos Naturales (13 de Junio de 2017). Secretaría de Medio Ambiente y Recursos Naturales. Recuperado el 3 de Agosto de 2018, de Restauración Forestal en la Comunidad Indígena de Cherán, en el Programa Forestal Federal Meseta Purépecha: https://www.gob.mx/cms/uploads/attachment/file/8544 0/Casos de exito Comunidad Indigena de Cheran.pdf

Svampa, M. (2012). Extractivismo neodesarrollista y movimientos sociales. ¿Un giro ecoterritorial hacia nuevas alternativas? En M. Lang y D. Mokrani (Coomp.), Más allá del desarrollo, 185-216. México: Fundación Rosa Luxemburgo/ Abya Yala

Svampa, M. (2013). Consenso de los Commodities y lenguajes de valoración en América Latina. Nueva Sociedad, 244, 30-46. Recuperado de https://nuso.org/articulo/consenso-de-los-commodities-y-lenguajes-de-valoracion-en-a merica-latina/

Tarrow, S. (1997). El Poder en Movimiento. Los Movimientos Sociales, la acción colectiva y la política. Madrid: Alianza Editorial.

Tarrow, S. (2002). Ciclos de acción colectiva: entre los momentos de locura y el repertorio de contestación. En M. Traugott (Coord.), Protesta social. Repertorios y Ciclos de acción colectiva. Barcelona: Editorial Hacer. 
Eika Cruz Coria, et al. La defensa de los recursos forestales: el movimiento social en la comunid...

Tarrow, S. (2004). El Poder en Movimiento. Los movimientos sociales, la acción colectiva y la política. España: Alianza Editorial.

Tilly, C. (1978). From mobilization to revolution. Michigan, EEUU: Addison-Wesley Publishing Company.

Touraine, A. (1995). Producción de la sociedad. México: ISS/UNAM/IFAL/Embajada de Francia.

Tourliere, M. (2017). El país, una gigantesca concesión (Mapas). Proceso. Recuperado de https://www.proceso.com. $\mathrm{mx} / 500030 /$ pais-una-gigantesca-concesion-mapas

Velázquez, J., y Lepe, L (2013). Parankuech, Diálogos y Aprendizajes: Las Fogatas de Cherán como praxis educativa comunitaria. International Journal of Multicultural Education, 15(3), 61-75, Recuperado de: https://go.gale.com/ps/anonymous?id=GALE\%7CA420198285\&sid=googleScholar\&v=2.1\&it=r\&link access $=$ abs\&issn $=19345267 \& \mathrm{p}=\mathrm{AONE} \& \mathrm{sw}=\mathrm{w}$

Ventura, M. (2012). Proceso de autonomía en Cherán. Movilizar el derecho. Espiral, Estudios sobre Estado y Sociedad, $X I X(55), 157-176$. Recuperado de: http://www.scielo.org.mx/scielo.php?script=sci_arttext\&pid=S1665-056 $\underline{52012000300006}$

\section{Notas}

1 Los movimientos sociales son un tipo de acción colectiva que a razón de su organización social buscan movilizar recursos en torno a intereses compartidos, presentan cierto nivel de organización, son acciones colectivas que se desarrollan y mantienen en el tiempo, y están orientados al cambio social. Estos elementos son los que, en gran medida, diferencian a los MS de otras formas de acción colectiva, por ejemplo, las demandas y las movilizaciones (Garretón, 2002).

\section{BY-NC-SA}

